Check for updates

Cite this: RSC Adv., 2019, 9, 3597

Received 13th August 2018

Accepted 14th January 2019

DOI: $10.1039 / \mathrm{c} 8 \mathrm{ra06797j}$

rsc.li/rsc-advances

\section{Controllable reduction of graphene oxide by electron-beam irradiation}

\author{
Yu Yang, ${ }^{a}$ Liang Chen, ${ }^{* b}$ De-Yuan Li, ${ }^{a}$ Ruo-Bing Yi, ${ }^{a}$ Jia-Wei Mo, ${ }^{b}$ Ming-Hong Wu${ }^{\star a}$ \\ and Gang Xu $\mathrm{DD}$ *a
}

The oxygen content of graphene oxide (GO) is directly related to its physical and chemical properties, such as hydrophilicity, suspension stability, adsorption, and ion-sieving ability of GO membranes. Here, a series of reduced $\mathrm{GO}(\mathrm{rGO})$ with $\mathrm{C} / \mathrm{O}$ atomic ratios from 1.6 to 4.8 were prepared conveniently by electron-beam irradiation (EBI) with irradiation-dose control. Moreover, a single oxygen-containing group, i.e., epoxy or carbonyl, could be retained mainly in the rGO. The interlayer spacing of rGO could be changed from 9.6 $\AA$ to $7.4 \AA$ through control of the oxygen content. The prepared rGO exhibited an excellent adsorption effect on $\mathrm{Pb}(\mathrm{I})$ ions, and the max adsorption capacity reached $194.76 \mathrm{mg} \mathrm{g}^{-1}$ for rGO with a low irradiation dose (5 kGy), which showed that the ratio of oxygen-containing groups is important for improving the adsorption of rGO in aqueous solution. These results indicated that highly efficient, environmentally friendly, and advanced EBI technology has good potential prospects for use in the large-scale production of rGO with precise control of the oxygen content.

\section{Introduction}

Graphene oxide (GO) and reduced graphene oxide (rGO) are two-dimensional (2D) materials derived from the oxidation of graphite, followed by one of many different possible exfoliation and reduction methods, and consequently have attracted great interest from researchers due to their unique physical and chemical characteristics. ${ }^{\mathbf{1}-4}$ They show potential for use in many diverse technology applications, such as ion sieves, ${ }^{\mathbf{1 , 5 , 6}}$ energy storage ${ }^{7-9}$ water treatment, ${ }^{\mathbf{1 0 , 1 1}}$ biomaterials ${ }^{\mathbf{1 2 , 1 3}}$ and conductive materials. $^{\mathbf{1 4 , 1 5}}$ The performance of these applications is closely related to the characteristics of GO and rGO, such as their conductivity, water stability, biocompatibility, specific surface area and adsorption performance. ${ }^{\mathbf{4}, 16}$ However, all these properties of GO are affected by the type and amount of oxygencontaining groups on the GO surface. Therefore, it is possible to obtain rGO with specific properties by reducing or controlling the oxygen-containing groups of GO. At present, the reduction methods of GO mainly focus on thermal reduction and chemical reductant methods, such as with hydrazine reductants, ${ }^{17}$ sodium borohydride reductants, ${ }^{18}$ ascorbic acid, ${ }^{19}$ amino acid, ${ }^{20}$ HI/HAC ${ }^{21}$ and other methods, including soft-lithography. ${ }^{22}$ However, these reduction methods have harsh reaction conditions (high temperatures for a long reaction time), introduce other impurities, and are typically not environmentally

${ }^{a}$ Shanghai Applied Radiation Institute, Shanghai University, Shanghai 200444, China. E-mail:mhwu@shu.edu.cn; xugang@shu.edu.cn

${ }^{b}$ Department of Optical Engineering, Zhejiang A\&F University, Lin'an, Zhejiang 311300, China. E-mail: liang_chen05@126.com friendly. ${ }^{4}$ Particularly important is to have a controllable oxygen content that can alter the properties of rGO to fulfil specific requirements in applications. There have also been efforts to tune the degrees of reduction. For example, it can be controlled by increasing the $\mathrm{C} / \mathrm{O}$ ratio in thermal reduction methods, ${ }^{23}$ which is not only temperature-dependent but also a timedependent process in the timeframe of seconds. So, due to the difficulty in precisely controlling the reduction process, ${ }^{6}$ a highly efficient and large-scale production of rGO has still not been achieved, especially, for reduction control, which still uses a higher oxygen content. Previous studies have demonstrated that GO has excellent adsorption for heavy metal ions and organic contaminants. ${ }^{24-27}$ However, swelling due to the strong hydrophilic character of GO membranes results in poor stability in water and hinders their application in water treatment. ${ }^{28}$

Electron-beam irradiation (EBI) is an advanced redox technique that has been used for synthesis and chemical modification of materials. ${ }^{29-31}$ The principle of this method is to get the active free radicals $\left(\cdot \mathrm{OH}, \cdot \mathrm{H}\right.$ and $\left.\mathrm{e}_{\mathrm{aq}}{ }^{-}\right)$to chemically react through applying high-energy-electron-beam radiation to water. So the strategy has many advantages, such as being chemicalreductant free, efficient, fast, eco-friendly, and easily scalable.

In this study, GO was reduced in a water/isopropanol solution system by simple, efficient, and fast EBI. The oxygen content in rGO decreased obviously as the reduction degree increased. Different reduction degrees of rGO with an interlayer spacing from 9.6 to $7.4 \AA$, and even a single oxygen-containing group, i.e., epoxy or carbonyl dominant, were obtained by controlling the EBI dose. Our previous studies also found that rGO as an ion-sieving membrane has great application in water 
treatment, and that different interlayer spacings can achieve accurate ion screening. ${ }^{1}$ Here, the results show that EBI can precisely control the rGO layer spacing under different irradiation doses, which further provides new ideas for controlling the layer spacing of rGO and the large-scale preparation of graphene. Moreover, rGO reduced by the EBI method showed a good adsorption of $\mathrm{Pb}(\mathrm{II})$ with different reduction degrees of rGO. It was found that a slight reduction of rGO at low doses (5 $\mathrm{kGy}$ ) resulted in the best adsorption of $\mathrm{Pb}(\mathrm{II})$ of $194.76 \mathrm{mg} \mathrm{g}^{-1}$. The use of rGO reduced by the EBI method with dose control not only solves the issue of the swelling of GO membranes in water treatment applications, but also allows achieving a good adsorption at the same time, such as of heavy metal ions.

\section{Experimental}

\section{Preparation of graphene oxide (GO) suspension}

Graphene oxide (GO) was prepared from commercial graphite powder via a modified Hummers method. ${ }^{32}$ Graphite powders were pre-oxidized by concentrated $\mathrm{H}_{2} \mathrm{SO}_{4}, \mathrm{~K}_{2} \mathrm{~S}_{2} \mathrm{O}_{8}$, and $\mathrm{P}_{2} \mathrm{O}_{5}$ solution with continuous stirring for several hours. Then, the mixture was washed with DI water and vacuum dried. Then, preoxidized graphite was further oxidized in concentrated $\mathrm{H}_{2} \mathrm{SO}_{4}$ and $\mathrm{KMnO}_{4}$, diluted with DI water, followed by the addition of $30 \% \mathrm{H}_{2} \mathrm{O}_{2}$. The product was separated by centrifugation and washed with a $1: 10 \mathrm{HCl}$ aqueous solution to remove ion species first, and then washed with DI water sequentially. The concentration of the as-prepared GO suspension was approximately $5 \mathrm{mg} \mathrm{ml}^{-1}$.

\section{Reduced graphene oxide (rGO) prepared by electron-beam irradiation (EBI)}

The EBI reduction method essentially involves applying radiation to water to produce hydrated electrons in the medium to facilitate chemical reactions. ${ }^{33,34}$ Hydrated electrons chemically react with the oxygen-containing groups on the GO surface to reduce the oxygen content. ${ }^{35}$ The intensity of the irradiation is described by the absorbed dose. The unit is grays, which are defined as the energy absorbed by the mass of the substance being irradiated: 1 gray indicates that $1 \mathrm{~kg}$ of material absorbs 1 $\mathrm{J}$ of energy, therefore, 1 gray $=1 \mathrm{~J} \mathrm{~kg}^{-1}$. Here the content of $75 \mathrm{mg}$ GO was irradiated in each of the suspension/isopropanol samples. The GO suspension and isopropanol were mixed at a volume ratio of $3: 2$. The mixed solutions were put into a sealed bag filled with nitrogen gas and irradiated with different doses $(0,5,15,20,40 \mathrm{kGy})$ under room temperature. The electron accelerator had a beam intensity of $5 \mathrm{~mA}$ and an energy of 1.8 MeV (From the Shanghai Institute of Radiation Applications, GJ-II type electron accelerator). The degree of reduction of rGO increased and the colour became darker with the gradual increase in the irradiation dose. These were due to the removal of some oxygen-containing groups and the decrease in hydrophilicity, which led to rGO sheet agglomeration. Finally, the rGO solution was washed sequentially with DI water by centrifugation.
In order to test the suspension stability of rGO reduced by the EBI, the prepared rGO suspensions with the radiation dose increasing were centrifuged at $5000 \mathrm{rpm}$ for $5 \mathrm{~min}$.

\section{Fabrication of freestanding rGO membranes}

Freestanding rGO membranes could be prepared by dropcasting the rGO suspension droplets onto a smooth paper substrate. ${ }^{1}$ The freestanding rGO membranes in our study were dried thoroughly at $60^{\circ} \mathrm{C}$ for $12 \mathrm{~h}$. After that, they were peeled off, rinsed, and soaked with DI water for more than half an hour, then dried in a dry dish at room temperature for 3 days. The prepared freestanding membranes were later used for the adsorption experiments.

\section{Adsorption experiments}

The kinetics of adsorption of $\mathrm{Pb}$ (II) by rGO was assessed by adding $25 \mathrm{mg}$ of rGO membranes in a $10 \mathrm{ml}$ solution with the initial concentration of $\mathrm{Pb}$ (II) $500 \mathrm{mg} \mathrm{l}^{-1}$, and the mixture was rotationally stirred at a rate of $160 \mathrm{rpm}$ at $30^{\circ} \mathrm{C}$. An aliquot of $0.2 \mathrm{ml}$ sample solution was taken from the beaker to analyze the concentration of lead ions at different time intervals. The concentration of $\mathrm{Pb}$ (II) ions was determined using ICP-OES (HITACHI, PS7800).

According to the following equation ${ }^{36,37}$ as a pseudo-firstorder kinetic model (eqn (1)), the experimental data were fitted using Origin software:

$$
q_{t}=q_{\mathrm{e}}\left(1-e^{-k_{1} t}\right)
$$

where $q_{\mathrm{e}}$ and $q_{t}(\mu \mathrm{g} / \mathrm{g})$ are the mass of antibiotics adsorbed per unit mass of adsorbent at equilibrium and at time $t(\mathrm{~h})$, respectively, and $k_{1}(1 / \mathrm{h})$ is the rate constant of the first-order kinetic model.

\section{Results and discussion}

\section{Theory of the reduction of GO suspension via electron-beam irradiation}

The primary radiolysis process of water under electron-beam irradiation can be explained by reaction $(1):^{33}$

$$
\begin{gathered}
\mathrm{H}_{2} \mathrm{O} \stackrel{\text { Electron beam }}{\longrightarrow} \cdot \mathrm{OH}, \cdot \mathrm{H}, \mathrm{e}_{\mathrm{aq}}{ }^{-}, \mathrm{H}_{2}, \mathrm{H}_{2} \mathrm{O}_{2}, \mathrm{H}_{3} \mathrm{O}^{+} \\
\mathrm{e}_{\mathrm{aq}}{ }^{-}+\cdot \mathrm{OH} \rightarrow \mathrm{OH}^{-} \\
\cdot \mathrm{OH}+\left(\mathrm{CH}_{3}\right)_{2} \mathrm{CHOH} \rightarrow \cdot \mathrm{C}\left(\mathrm{CH}_{3}\right)_{2} \mathrm{OH}+\mathrm{H}_{2} \mathrm{O} \\
\mathrm{e}_{\mathrm{aq}}{ }^{-}+\cdot \mathrm{C}\left(\mathrm{CH}_{3}\right)_{2} \mathrm{OH} \rightarrow \mathrm{C}\left(\mathrm{CH}_{3}\right)_{2} \mathrm{OH}^{-}
\end{gathered}
$$

In reaction (1), water molecules produce various species under the high-energy-electron-beam irradiation, where the hydrated electron $\left(\mathrm{e}^{-}+n \mathrm{H}_{2} \mathrm{O} \rightarrow \mathrm{e}_{\mathrm{aq}}{ }^{-}\right)$is a strong reducing agent toward oxygen-containing functional groups, such as hydroxyl, epoxies, ketones, and carboxylic acids. The generated $\mathrm{e}_{\mathrm{aq}}{ }^{-}$can cause the deoxygenation and hence reduction of GO. However, $\mathrm{e}_{\mathrm{aq}}{ }^{-}$tend to be degraded by $\cdot \mathrm{OH}$ in reaction (2). This causes the reduction reaction to be blocked, thus it is 
a

C

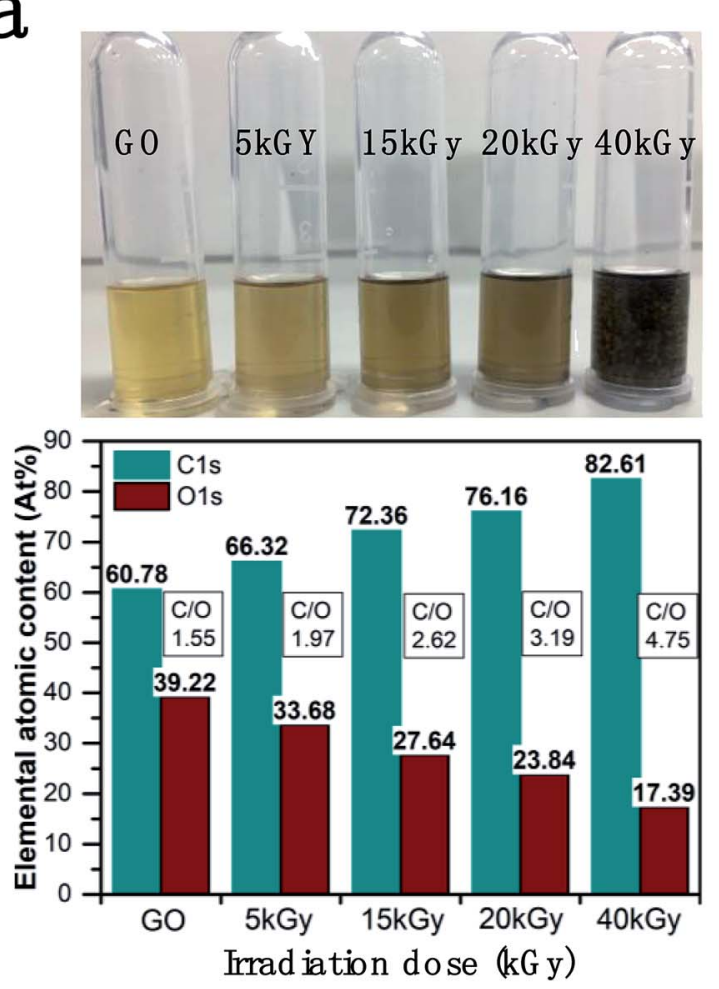

b
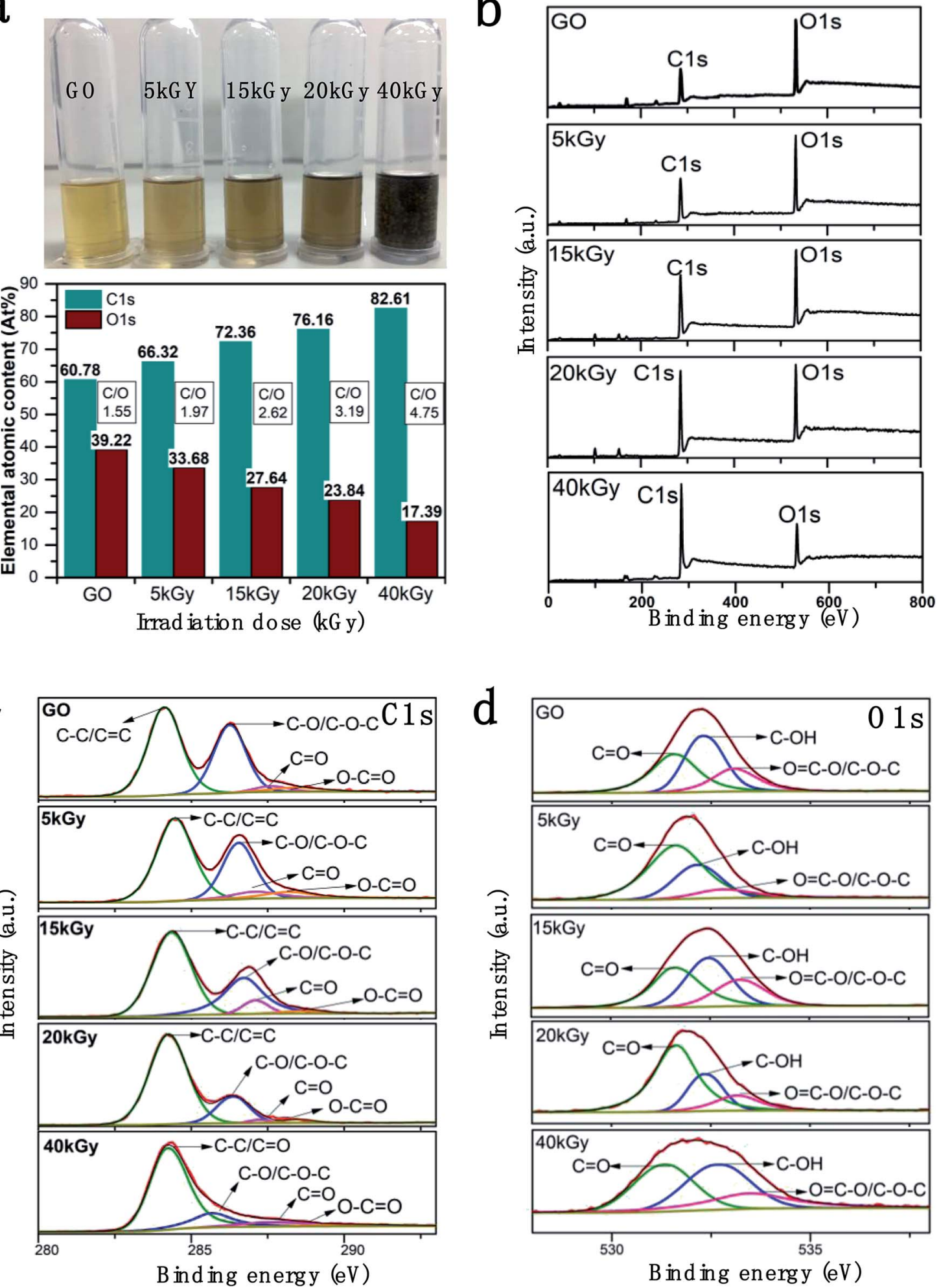

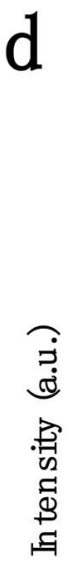
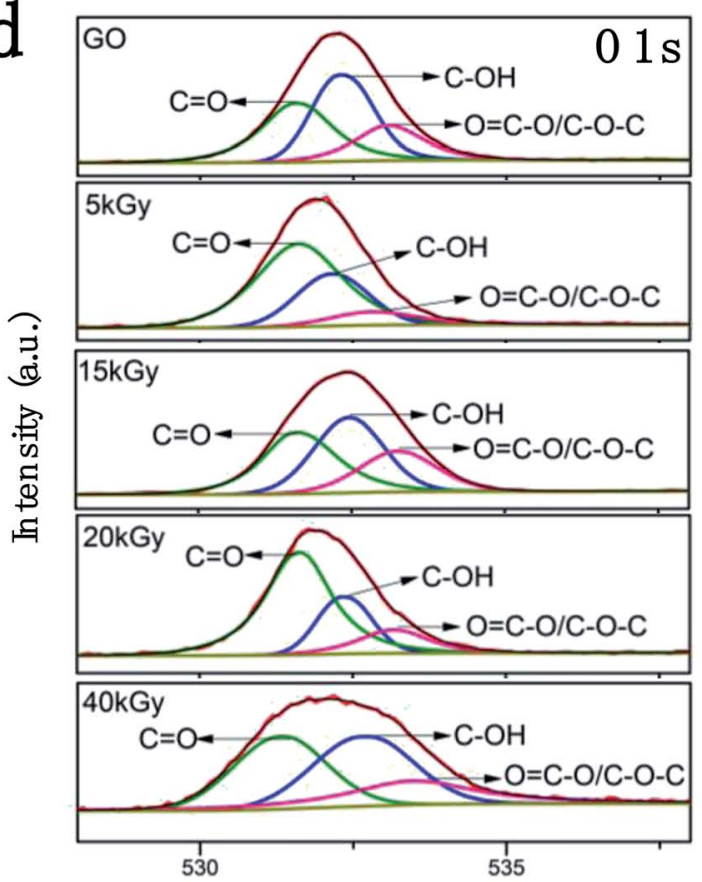

$\mathrm{B}$ ind ing energy $(\mathrm{eV})$

Fig. 1 X-ray photoelectron spectrometer spectra (XPS) of the rGOs: (a) GO and rGOs solution $\left(0.2 \mathrm{mg} \mathrm{ml}^{-1}\right)$ irradiated by an electron beam at different irradiation doses and elemental atomic content (at.\%) of C/O; (b) full-scan spectra of the samples of GO and rGOs irradiated to different doses; (c) C1s peak; (d) O1s peak.

necessary to add an alcohol to the water to scavenge the hydroxyl radicals $(\cdot \mathrm{OH})$ to prevent reaction $(2)$, at the same time greatly enhancing the stability of the hydrated electron by reaction (3). Herein, it is assumed that reaction (4) does not occur to an appreciable extent. ${ }^{34}$ 


\section{Physicochemical properties of GO and rGOs}

The rGOs solution $\left(0.2 \mathrm{mg} \mathrm{m} \mathrm{ml}^{-1}\right)$ at different irradiation doses $(0,5,15,20,40 \mathrm{kGy})$ were obtained as shown in Fig. 1a. The solution became darker with increasing the irradiation dose, which was consistent with rGO dispersion reported in the literature. ${ }^{38}$ The decrease in GO hydrophilicity due to removing a portion of the oxygen-containing groups, which are easier to be separated by centrifugation, results in the agglomeration of GO sheets.

X-ray photoelectron spectroscopy (XPS) was utilized to analyze the oxygen group content before and after the irradiation of GO. The XPS full scan spectra and C1s and O1s partial spectra of rGOs under different irradiation doses are shown in Fig. 1. The C1s and O1s peak positions of rGOs were at $285.08 \mathrm{eV}$ and $532.08 \mathrm{eV}$, respectively. From the elemental contents of $\mathrm{C}$ and $\mathrm{O}$ in the full scan spectra, it is obvious that the ratio of $\mathrm{C} / \mathrm{O}$ in each sample increased sharply (from 1.6 to 4.8 ) with the increase in irradiation dose, indicating the successful and controllable removal of oxygen-containing groups on the GO surface. The C1s and O1s XPS spectra of rGOs with different irradiation dosages are shown in Fig. 1c and d. The C1s XPS spectra exhibit four different peaks located at 284.46, 286.54, 287.08 and $288.13 \mathrm{eV}$, corresponding to aromatic $(\mathrm{C}-\mathrm{C} / \mathrm{C}=\mathrm{C})$, epoxide $(\mathrm{C}-\mathrm{O} / \mathrm{C}-\mathrm{O}-\mathrm{C})$, carbonyl $(\mathrm{C}=\mathrm{O})$ and carboxyl group $(\mathrm{O}-$ $\mathrm{C}=\mathrm{O}$ ) chemical shifts, respectively. ${ }^{1,39} \mathrm{~A}$ clear reduction control is shown by the specific peak area of $\mathrm{C}-\mathrm{O} / \mathrm{C}-\mathrm{O}-\mathrm{C}$ decreasing with the increasing irradiation dosages, as shown in Fig. 1c. The content of $\mathrm{C}-\mathrm{O} / \mathrm{C}-\mathrm{O}-\mathrm{C}$ decreased from $39.11 \%$ to $11.07 \%$, and the content of $\mathrm{O}=\mathrm{C}-\mathrm{O}$ group was reduced from $88.75 \%$ to $25.58 \%$ with an increase in irradiation dose. On the contrary, the proportion of $\mathrm{C}-\mathrm{C} / \mathrm{C}=\mathrm{C}$ gradually increased from $52.73 \%$ to $80.55 \%$. (Table 1). Similarly, the O1s spectrum was also resolved into three individual component peaks centred at $533.16 \mathrm{eV}$ $(\mathrm{O}=\mathrm{C}-\mathrm{O}$ and $\mathrm{C}-\mathrm{O}-\mathrm{C}), 532.32 \mathrm{eV}(\mathrm{C}-\mathrm{OH})$ and $531.60 \mathrm{eV}(\mathrm{C}=$ $\mathrm{O}),{ }^{\mathbf{4 0 , 4 1}}$ respectively (Fig. 1d). The content of $\mathrm{C}-\mathrm{OH}$ decreased from $42.42 \%$ to $26.24 \%$, and that of $\mathrm{COOH}$ decreased from $20.31 \%$ to $12.16 \%$ (Table 2), which were consistent with the results for C1s XPS. This indicated that a large number of oxygen-containing groups had been removed as the irradiation dose increased. The main oxygen-containing groups retained in rGO were epoxy or carbonyl, which upon reduction with high irradiation doses were reduced to epoxy $\sim 11.07 \%$ and carbonyl $\sim 8.02 \%$ (total oxygen content was $\sim 17.2 \%$ at $40 \mathrm{kGy}$ ).

We confirmed the controllable reduction of oxygencontaining groups using FT-IR spectra detection, which

Table 1 C1s XPS elemental analysis data of the samples at different irradiation doses

\begin{tabular}{lllll}
\hline $\begin{array}{l}\text { Irradiation } \\
\text { dose (kGy) }\end{array}$ & $\mathrm{C}-\mathrm{C} / \mathrm{C}=\mathrm{C}(\%)$ & $\mathrm{C}-\mathrm{O} / \mathrm{C}-\mathrm{O}-\mathrm{C}(\%)$ & $\mathrm{C}=\mathrm{O}(\%)$ & $\mathrm{O}-\mathrm{C}=\mathrm{O}(\%)$ \\
\hline $\mathrm{GO}(0 \mathrm{kGy})$ & 52.73 & 39.1 & 5.18 & 2.99 \\
$5 \mathrm{kGy}$ & 56.79 & 35.12 & 6.51 & 1.58 \\
$15 \mathrm{kGy}$ & 61.05 & 30.19 & 6.46 & 2.31 \\
$20 \mathrm{kGy}$ & 75.42 & 21.43 & 1.33 & 1.82 \\
$40 \mathrm{kGy}$ & 80.55 & 11.07 & 8.02 & 0.37
\end{tabular}

Table 2 O1s XPS elemental analysis data of the samples at different irradiation doses

\begin{tabular}{llll}
\hline $\begin{array}{l}\text { Irradiation } \\
\text { dose }(\mathrm{kGy})\end{array}$ & $\mathrm{C}=\mathrm{O}(\%)$ & $\mathrm{C}-\mathrm{OH}(\%)$ & $\mathrm{O}-\mathrm{C}=\mathrm{O} / \mathrm{C}-\mathrm{O}-\mathrm{C}(\%)$ \\
\hline $\mathrm{GO}(0 \mathrm{kGy})$ & 37.28 & 42.42 & 20.31 \\
$5 \mathrm{kGy}$ & 59.68 & 29.29 & 11.03 \\
$15 \mathrm{kGy}$ & 53.94 & 20.41 & 26.65 \\
$20 \mathrm{kGy}$ & 60.88 & 25.47 & 13.65 \\
$40 \mathrm{kGy}$ & 61.59 & 26.24 & 12.16
\end{tabular}

showed the same trends as the oxygen-containing group contents with the irradiation doses increasing. Fig. 2 shows the change in different oxygen-containing groups on the graphene oxide surface. This suggests that the oxygen-containing groups $-\mathrm{OH}\left(3430 \mathrm{~cm}^{-1}\right)$, carboxyl $\mathrm{C}=\mathrm{O}\left(1735 \mathrm{~cm}^{-1}\right)$, hydroxyl $\mathrm{C}-\mathrm{OH}$ $\left(1416 \mathrm{~cm}^{-1}\right)$, epoxy $\mathrm{C}-\mathrm{O}-\mathrm{C}\left(1240 \mathrm{~cm}^{-1}\right)$ and alkoxy $\mathrm{C}-\mathrm{O}$ $\left(1074 \mathrm{~cm}^{-1}\right)$ were all gradually decreased with the radiation dose increasing. At $40 \mathrm{kGy}$ dose irradiation, the oxygencontaining groups almost disappeared, such as the $-\mathrm{OH}$ (3430 $\left.\mathrm{cm}^{-1}\right)$, carbonyl $\mathrm{C}=\mathrm{O}\left(1735 \mathrm{~cm}^{-1}\right)$, carbonyl $\mathrm{C}-\mathrm{OH}$ $\left(1416 \mathrm{~cm}^{-1}\right)$ and alkoxy $\mathrm{C}-\mathrm{O}\left(1074 \mathrm{~cm}^{-1}\right)$ groups, consistent with the reduction of rGO reported in the literature. ${ }^{42-48}$ Moreover, a single and less oxygen-containing group, such as epoxy or carbonyl, can be retained in rGO. Therefore, it was indicated that a series of different degrees of reduction of rGO were successfully obtained by electron-beam irradiation.

Raman spectroscopy is a non-destructive technique used to study the structural orders and disorders of various carbon materials, including GO and rGO. ${ }^{49}$ The Raman spectra of GO and rGO in Fig. 3b show peculiar peaks designated as a D band at $1350 \mathrm{~cm}^{-1}$ and $\mathrm{G}$ band at $1590 \mathrm{~cm}^{-1}$, with the $\mathrm{G}$ band assigned to the first-order scattering of the $\mathrm{E}_{2 \mathrm{~g}}$ mode observed for $\mathrm{sp}^{2}$ carbon domains, and the broad $\mathrm{D}$ band caused by $\mathrm{sp}^{3}$ hybridized carbon, structural defects, carbon amorphous or edge planes that can break the symmetry and selection rule, The $I_{\mathrm{D}} / I_{\mathrm{G}}$ ratio is usually used to measure the disorder degree and

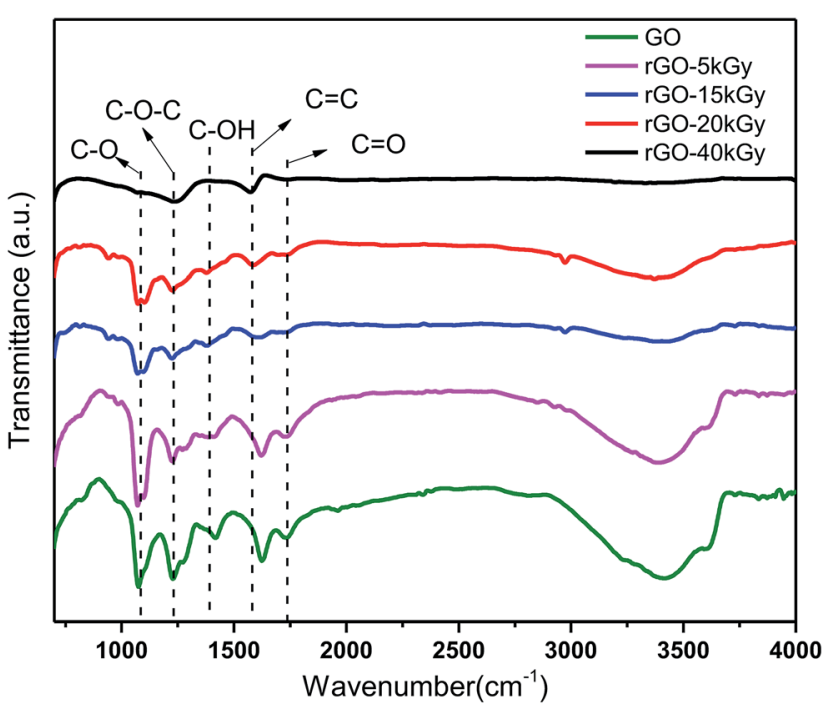

Fig. 2 FTIR spectra of rGO under different irradiation doses $(0,5,15$, 20, 40 kGy). 
a

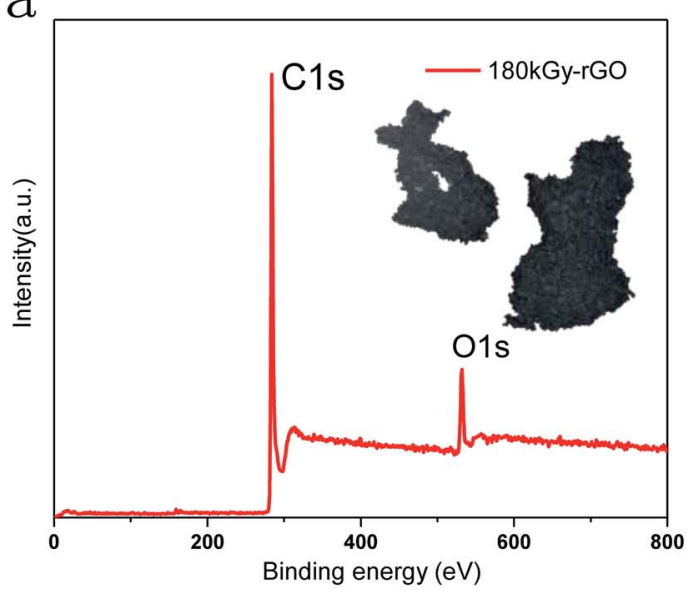

$\mathrm{b}$

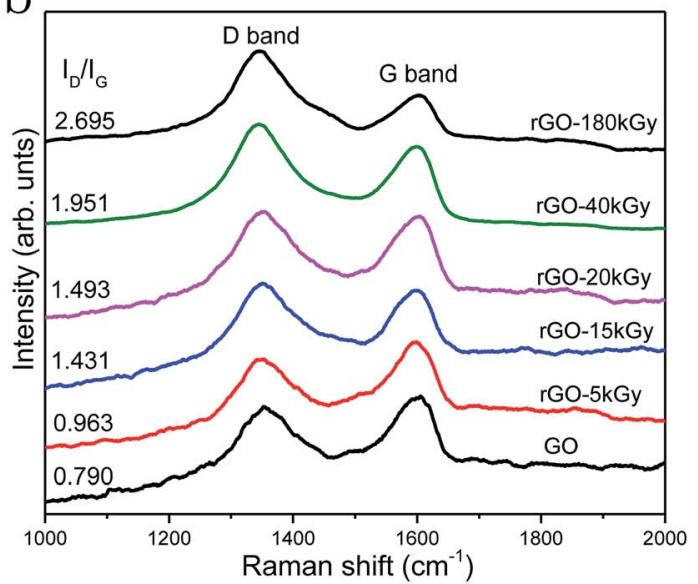

Fig. 3 X-ray photoelectron spectrometer spectra (XPS) and Raman spectra of the rGOs. (a) XPS of rGO irradiated with 180 kGy. Inset shows a photo of agglomerated rGOs; (b) Raman spectra of the rGOs irradiated with different irradiation doses.

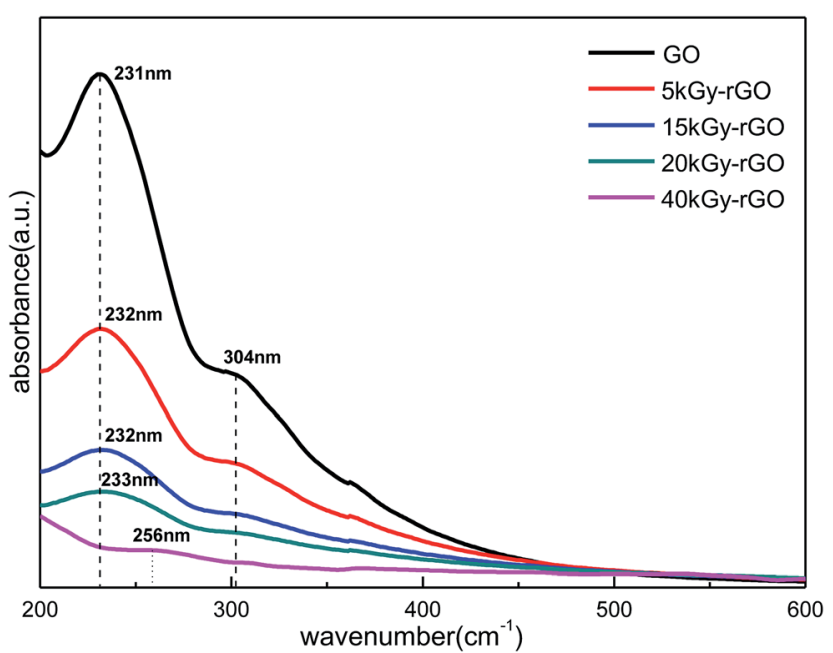

Fig. 4 UV-visible absorption spectra of GO and rGOs.

size of $\mathrm{sp}^{2}$ clusters in a network of $\mathrm{sp}^{3}$ and $\mathrm{sp}^{2}$ bonded carbons. ${ }^{50,51} \mathrm{An}$ increase in the $I_{\mathrm{D}} / I_{\mathrm{G}}$ value means a degradation of the crystallinity of the graphitic materials. Here, the Raman spectroscopy results (Fig. 3) showed that the $I_{\mathrm{D}} / I_{\mathrm{G}}$ value increased from 0.790 to 1.951 with the increase in irradiation dose, thereby the defects had increased following electronbeam irradiation.

To analyze the oxygen content and defects in GO at high doses, the irradiation dose was increased to $180 \mathrm{kGy}$. According to the results of XPS and Raman detection, the $\mathrm{C}-\mathrm{C} / \mathrm{C}=\mathrm{C}$ ration increased to $91.28 \%$, and the corresponding defects also increased significantly, as shown in Fig. 3. These indicate that there was a higher reduction with the increased irradiation doses; however, the defects and agglomeration of rGO was a serious issue (inset in Fig. 3a), and further confirmed that a controllable oxygen content of rGO is important to fulfil specific requirements in applications.

Fig. 4 shows the typical UV-vis absorption spectra for GO and rGO. There are two distinct characteristic absorption peaks at $231 \mathrm{~nm}$ and $304 \mathrm{~nm}$ for GO (0 kGy), which correspond to the $\pi$ $\rightarrow \pi^{*}$ transition of $\mathrm{C}=\mathrm{C}$ and the $\mathrm{n} \rightarrow \pi^{*}$ transition of $\mathrm{C}=\mathrm{O}$, respectively. ${ }^{52}$ With the increase in radiation dose (from 0 to 40 $\mathrm{kGy}$ ), the absorption peak of GO at $231 \mathrm{~nm}$ was shifted slightly to a longer wavelength near $256 \mathrm{~nm}$. This shows that the conjugated electron structure of graphene oxide is gradually
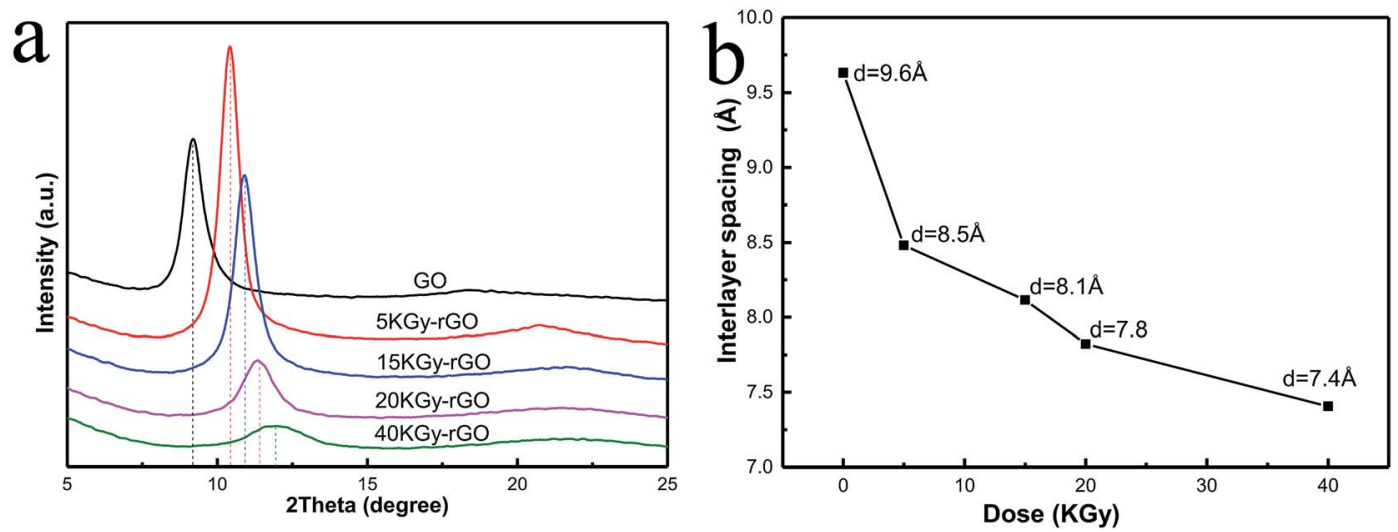

Fig. 5 (a) XRD spectra of rGOs and (b) interlayer spacing of $r G O$ under different irradiation doses $(0,5,15,20,40$ kGy). 


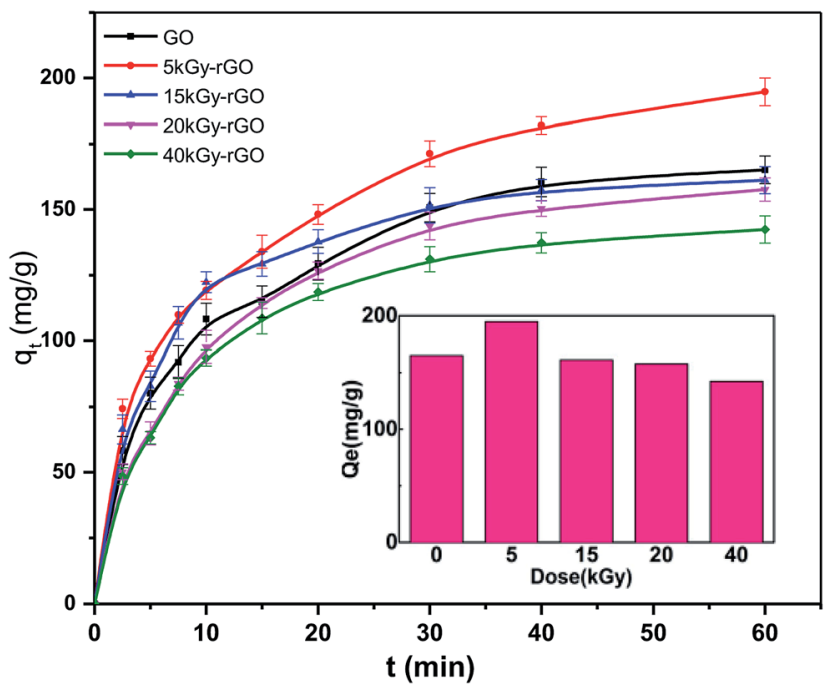

Fig. 6 Adsorption kinetics of $\mathrm{Pb}(\Perp)$ on $\mathrm{GO}$ and $\mathrm{rGO}$ under different irradiation doses $(0,5,15,20,40 \mathrm{kGy})$. The inset chart shows the relationship between rGO with different irradiation doses and the adsorption capacity of $\mathrm{Pb}(॥)$.

restored, which is an indication of the increase in GO reduction. The significant decrease of the $\mathrm{C}=\mathrm{O}$ characteristic absorption peak at $304 \mathrm{~nm}$ (disappears at $40 \mathrm{kGy}$ ) further indicated a decrease in the GO oxygen content. These results demonstrate again that a series of different degrees of reduction of rGO could be obtained by precise control of the EBI.

The control or tuning of the interlayer spacing is a key issue for ion sieving by GO membranes. ${ }^{1,5}$ It is obvious that, the oxygencontaining groups serving as pillars supporting the spacing between GO flakes in membranes would have an impact on the interlayer spacing. We therefore tested the interlayer spacing of fabricated rGO membranes by X-ray diffractometry (XRD), and found that there were clear shifts of the spacing (indicated by the Bragg peaks in Fig. 5) relative to the GO membranes without EBI reduction. The interlayer spacing showed a typical XRD pattern of the rGO, with spacings of 9.6, 8.5, 8.1, 7.8 and 7.4 ̊̊ for irradiated doses of $0,5,15,20$ and $40 \mathrm{kGy}$, respectively. As the radiation dose increased, the interlayer spacing of rGO decreased from 9.6 $\AA$ to $7.4 \AA$. This shows that the oxide contents can determine the interlayer spacing of rGO membranes at sizes as small as a nanometre, and the variable range of this spacing can be controlled to within $1 \AA$. The decrease in interlayer spacing indicated that EBI could precisely deoxygenate and control the reduction of rGO. It should be noted that the higher radiation dose resulted in a weaker intensity of XRD peaks (Fig. 5a), indicating a weakening of the levelling and ordering between GO flakes by higher EBI doses, which would decrease the permeability and mechanical properties of the membranes.

In addition, we analyzed the solution adsorption of the rGO membranes with heavy metal ions. The adsorption

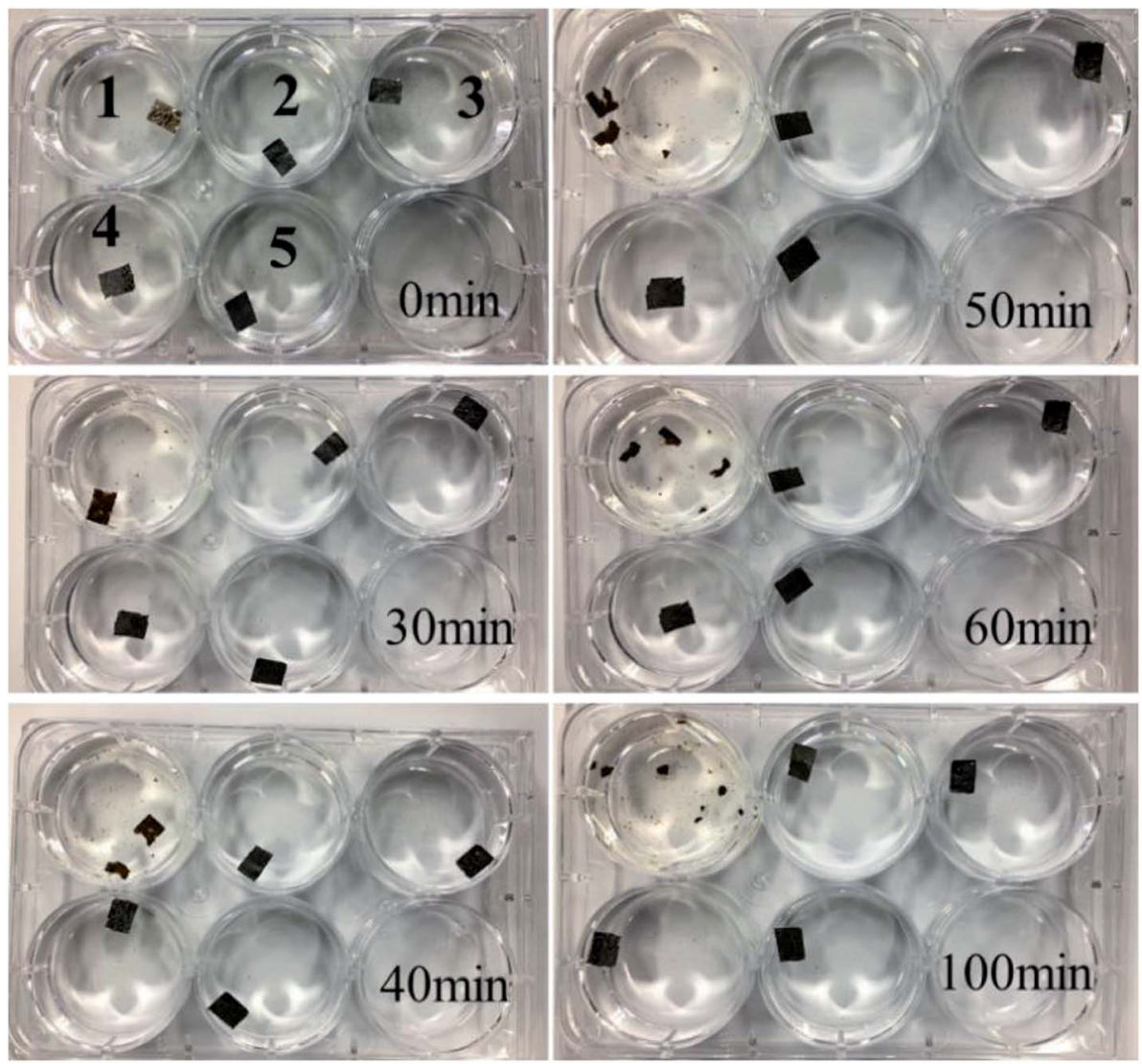

Fig. 7 Drastically different stability of GO and rGO membranes in water. (1): GO, (2): rGO (5 kGy), (3): rGO (15 kGy), (4): rGO (20 kGy) and (5): rGO (40 kGy). The photos were taken after the solutions had been stirred with a glass stirring rod. 
performance of $\mathrm{Pb}$ (II) by rGO membranes with different degrees of reduction was measured as shown in Fig. 6. It was found that a rapid adsorption process took place in the first $40 \mathrm{~min}$ and reached an equilibrium of adsorption at $\sim 1 \mathrm{~h}$. This rapid adsorption phenomenon is attributed to the strong cation- $\pi$ interactions between the adsorbents and heavy metal ions, which allows a large number of heavy metal ions to enter the channel between layers to achieve adsorption equilibrium. ${ }^{53}$ The adsorption capacity of $\mathrm{Pb}$ (II) by rGO under the different doses was also studied. The results showed that the fastest adsorption rate and maximum equilibrium adsorption capacity (194.76 $\mathrm{mg} \mathrm{g}^{-1}$ ) occurred at a low dose of $5 \mathrm{kGy}$. According to previous research, the mechanism of rGO adsorption of heavy metals is mainly dominated by cation- $\pi$ interactions, and the intensity of the cation- $\pi$ force is related to the area of the aromatic ring. However, the area of the aromatic ring is related to the hydrophilicity of rGO, and these are determined by the oxygen contents on the rGO surface. ${ }^{54}$ That is, aromatic rings are the main contributing factor for $\mathrm{Pb}$ adsorption, but also have an important influence on the hydrophilicity. Therefore, the reason for this phenomenon is that the GO reduced by the 5 kGy dose both had a certain reducibility with good stability and good hydrophilicity in water. Then, when the irradiation dose was higher than $5 \mathrm{kGy}$, the maximum adsorption capacity decreased with the increase in irradiation dose. This is related to the removal of oxygen-containing groups on the surface of the rGO.

Further experiments were carried out to observe the stability of GO and rGO membranes. As shown in Fig. 7, the most striking difference between the GO and rGO membranes is their stability in water, which shows that the GO membrane disintegrates after about $30 \mathrm{~min}$ upon hydration without any mechanical agitation, and is then completely dispersed after $100 \mathrm{~min}$. In contrast, all the rGO membranes remained intact in water. This indicated that the reduction of GO by EBI, to a certain extent, can well solve the swelling of GO membranes in water.

\section{Conclusions}

We achieved a facile and precise control of the oxygen content in $\mathrm{rGO}$, with $\mathrm{C} / \mathrm{O}$ atomic ratios from 1.6 to 4.8 , and retention of a single epoxy or carbonyl group, mainly though electron-beam irradiation at different irradiation doses (5, 15, 20, $40 \mathrm{kGy})$. Under the electron-beam irradiation, a large number of oxygen functional groups on the surface of the GO were controllably removed, and the interlayer spacing of GO decreased from 9.6 to $7.4 \AA$ A, showing that the oxide content can determine the interlayer spacing of rGO membranes at sizes as small as a nanometre; also, the variable range of this spacing could be controlled to within $1 \AA$. The adsorption performance of $\mathrm{Pb}$ (II) showed that the best adsorption effect occurred when the irradiation dose was $5 \mathrm{kGy}$, which represented the fastest adsorption rate and maximum equilibrium adsorption capacity (194.76 $\mathrm{mg} \mathrm{g}^{-1}$ ). Overall, our findings represent a controllable reduction of GO by EBI methods as an environmentally friendly, efficient and advanced technology that has potential application prospects in the controllable reduction of GO, energy storage, ion sieves, water treatment, biomaterials, conductive materials, among others.

\section{Conflicts of interest}

There are no conflicts to declare.

\section{Acknowledgements}

This work was supported by the National Natural Science Foundation of China (no. 11675098, 21672231, 11605111, 11775138), the Scientific Research and Developed Fund of Zhejiang A \& F University (no. 2017FR032).

\section{References}

1 L. Chen, G. Shi, J. Shen, B. Peng, B. Zhang, Y. Wang, F. Bian, J. Wang, D. Li, Z. Qian, G. Xu, G. Liu, J. Zeng, L. Zhang, Y. Yang, G. Zhou, M. Wu, W. Jin, J. Li and H. Fang, Nature, 2017, 550, 380-383.

2 D. R. Dreyer, S. Park, C. W. Bielawski and R. S. Ruoff, Chem. Soc. Rev., 2010, 39, 228-240.

3 Y. Zhu, S. Murali, W. Cai, X. Li, J. W. Suk, J. R. Potts and R. S. Ruoff, Adv. Mater., 2010, 22, 3906-3924.

4 S. Pei and H.-M. Cheng, Carbon, 2012, 50, 3210-3228.

5 J. Abraham, K. S. Vasu, C. D. Williams, K. Gopinadhan, Y. Su, C. T. Cherian, J. Dix, E. Prestat, S. J. Haigh, I. V. Grigorieva, P. Carbone, A. K. Geim and R. R. Nair, Nat. Nanotechnol., 2017, 12, 546-550.

6 R. K. Joshi, P. Carbone, F. C. Wang, V. G. Kravets, Y. Su, I. V. Grigorieva, H. A. Wu, A. K. Geim and R. R. Nair, Science, 2014, 343, 752-754.

7 J. Xu, K. Wang, S.-Z. Zu, B.-H. Han and Z. Wei, ACS Nano, 2010, 4, 5019-5026.

8 T. Kuila, A. K. Mishra, P. Khanra, N. H. Kim and J. H. Lee, Nanoscale, 2013, 5, 52-71.

9 Z. Bo, X. Shuai, S. Mao, H. Yang, J. Qian, J. Chen, J. Yan and K. Cen, Sci. Rep., 2014, 4, 4684.

10 V. Kumar Gupta, S. Agarwal, M. Asif, A. Fakhri and N. Sadeghi, J. Colloid Interface Sci., 2017, 497, 193-200.

11 W. Hou, Y. Zhang, T. Liu, H. Lu and L. He, $R S C A d v$., 2015, 5, 8037-8043.

12 B. C. Thompson, E. Murray and G. G. Wallace, Adv. Mater., 2015, 27, 7563-7582.

13 D. Ege, A. R. Kamali and A. R. Boccaccini, Adv. Eng. Mater., 2017, 19, 1700627.

14 A. T. U. Nugrahenny, J. Kim, S.-K. Kim, D.-H. Peck, S.-H. Yoon and D.-H. Jung, Carbon Lett., 2014, 15, 38-44.

15 C. Zhang, T. Zhai, Y. Dan and L.-S. Turng, Polym. Compos., 2017, 38, E199-E207.

16 Y. H. Xi, J. Q. Hu, Z. Liu, R. Xie, X. J. Ju, W. Wang and L. Y. Chu, ACS Appl. Mater. Interfaces, 2016, 8, 15557-15566.

17 S. Park, J. An, J. R. Potts, A. Velamakanni, S. Murali and R. S. Ruoff, Carbon, 2011, 49, 3019-3023. 
18 H.-J. Shin, K. K. Kim, A. Benayad, S.-M. Yoon, H. K. Park, I.-S. Jung, M. H. Jin, H.-K. Jeong, J. M. Kim, J.-Y. Choi and Y. H. Lee, Adv. Funct. Mater., 2009, 19, 1987-1992.

19 J. Zhang, H. Yang, G. Shen, P. Cheng, J. Zhang and S. Guo, Chem. Commun., 2010, 46, 1112-1114.

20 D. Chen, L. Li and L. Guo, Nanotechnology, 2011, 22, 325601.

21 Z. Xiong, C. Liao, W. Han and X. Wang, Adv. Mater., 2015, 27, 4469-4475.

22 Z. Xiong, C. Liao and X. Wang, J. Mater. Chem. C, 2015, 3, 6224-6231.

23 C. Punckt, F. Muckel, S. Wolff, I. A. Aksay, C. A. Chavarin, G. Bacher and W. Mertin, Appl. Phys. Lett., 2013, 102, 023114.

24 G. Zhao, X. Ren, X. Gao, X. Tan, J. Li, C. Chen, Y. Huang and X. Wang, Dalton Trans., 2011, 40, 10945-10952.

25 C. J. Madadrang, H. Y. Kim, G. Gao, N. Wang, J. Zhu, H. Feng, M. Gorring, M. L. Kasner and S. Hou, ACS Appl. Mater. Interfaces, 2012, 4, 1186-1193.

26 Y. Lei, F. Chen, Y. Luo and L. Zhang, Chem. Phys. Lett., 2014, 593, 122-127.

27 B. Peng, L. Chen, C. Que, K. Yang, F. Deng, X. Deng, G. Shi, G. Xu and M. Wu, Sci. Rep., 2016, 6, 31920.

28 P. Sun, K. Wang and H. Zhu, Adv. Mater., 2016, 28, 22872310.

29 E. Jin, J. He, K. Sheng, Z. Zhang, G. Shi and Q. Zheng, Acta Mater., 2013, 61, 6466-6473.

30 T. Sugimoto and K. Kimura, Bull. Chem. Soc. Jpn., 2013, 86, 333-338.

31 M. Kang, D. H. Lee, Y.-M. Kang and H. Jung, Electrochim. Acta, 2015, 184, 427-435.

32 W. S. Hummers and R. E. Offeman, J. Am. Chem. Soc., 1958, 80, 1339.

33 W. H. Hamill, J. Phys. Chem., 1969, 73, 1341-1347.

34 J. Cygler and G. R. Freeman, Can. J. Chem., 1984, 62, 12651270.

35 B. Zhang, L. Li, Z. Wang, S. Xie, Y. Zhang, Y. Shen, M. Yu, B. Deng, Q. Huang, C. Fan and J. Li, J. Mater. Chem., 2012, 22, 7775 .

36 Y. S. Ho, J. C. Y. Ng and G. McKay, Sep. Purif. Methods, 2000, 29, 189-232.
37 R. Ocampo-Perez, J. Rivera-Utrilla, C. Gomez-Pacheco, M. Sanchez-Polo and J. J. Lopez-Penalver, Chem. Eng. J., 2012, 213, 88-96.

38 J.-M. Jung, C.-H. Jung, M.-S. Oh, I.-T. Hwang, C.-H. Jung, K. Shin, J. Hwang, S.-H. Park and J.-H. Choi, Mater. Lett., 2014, 126, 151-153.

39 J. Bai, H. Sun, X. Yin, X. Yin, S. Wang, A. E. Creamer, L. Xu, Z. Qin, F. He and B. Gao, ACS Appl. Mater. Interfaces, 2016, 8, 25289-25296.

40 Z. Huang, Z. Li, L. Zheng, L. Zhou, Z. Chai, X. Wang and W. Shi, Chem. Eng. J., 2017, 328, 1066-1074.

41 I. Bertóti, M. Mohai and K. László, Carbon, 2015, 84, 185196.

42 Y. Shen, H.-B. Zhang, H. Zhang, W. Ren, A. Dasari, G.-S. Tang and Z.-Z. Yu, Carbon, 2013, 56, 132-138.

43 E. Fuente, J. A. Menéndez, M. A. Díez, D. Suárez and M. A. Montes-Morán, J. Phys. Chem. B, 2003, 107, 6350-6359.

44 T. Szabó, O. Berkesi and I. Dékány, Carbon, 2005, 43, 31863189.

45 T. Szabo, O. Berkesi, P. Forgo, K. Josepovits, Y. Sanakis, D. Petridis and I. Dekany, Chem. Mater., 2006, 18, 2740-2749.

46 A. Bagri, C. Mattevi, M. Acik, Y. J. Chabal, M. Chhowalla and V. B. Shenoy, Nat. Chem., 2010, 2, 581.

47 C.-J. Kim, W. Khan and S.-Y. Park, Chem. Phys. Lett., 2011, 511, 110-115.

48 C. Zhang, D. M. Dabbs, L.-M. Liu, I. A. Aksay, R. Car and A. Selloni, J. Phys. Chem. C, 2015, 119, 18167-18176.

49 A. Thakur, S. Kumar and V. S. Rangra, in Proceedings of the International Conference on Condensed Matter Physics 2014, ed. R.Sharma, P. K.Ahluwalia and M.Singh, 2015, vol. 1661.

50 M. Zainy, N. M. Huang, S. Vijay Kumar, H. N. Lim, C. H. Chia and I. Harrison, Mater. Lett., 2012, 89, 180-183.

51 A. Kaniyoor and S. Ramaprabhu, AIP Adv., 2012, 2, 13.

52 J. Li, G. Xiao, C. Chen, R. Li and D. Yan, J. Mater. Chem. A, 2013, 1, 1481-1487.

53 V. Chandra and K. S. Kim, Chem. Commun., 2011, 47, 39423944.

54 G. Shi, J. Liu, C. Wang, B. Song, Y. Tu, J. Hu and H. Fang, Sci. Rep., 2013, 3, 3436. 